\title{
Erosion of bowel by catheter of peritoneal access device during intraperitoneal chemotherapy: A case report and PRISMA-driven systematic review
}

\author{
Hung-Hsin Lin ${ }^{1,2 \#}$, Hou-Hsuan Cheng ${ }^{1,3 \#}$, Yi Chang ${ }^{1,4}$, Yen-Hou Chang ${ }^{1,4}$ and Chi-Mu Chuang1,4* \\ ${ }^{1}$ Institute of Clinical Medicine, School of Medicine, National Yang-Ming University, Taipei, Taiwan \\ ${ }^{2}$ Division of Colon \& Rectal Surgery, Department of Surgery, Taipei Veterans General Hospital, Taiwan \\ ${ }^{3}$ Division of Colorectal Surgery, Department of Surgery, Taichung Veterans General Hospital, Taiwan \\ ${ }^{4}$ Section of Gynecologic Oncology, Department of Obstetrics and Gynecology, Taipei Veterans General Hospital, Taipei, Taiwan \\ \#Author contributed equally to this work
}

\begin{abstract}
Several randomized trials have proved that intraperitoneal chemotherapy can confer better overall survival in the treatment of epithelial ovarian cancer. Also, intraperitoneal chemotherapy has been proved to be an effective alternative treatment for colorectal peritoneal carcinomatosis in non-randomized trials. However, complications induced by intraperitoneal chemotherapy deserve attention because of potential mortality in severe relevant cases. Commonly seen complications include infection, obstruction to infusion, retraction, leakage of infusion solution, and bowel erosion by catheter of peritoneal access device. Among these complications, bowel erosion is a relatively uncommon complication with reported rate between $1.2-4.2 \%$. Nonetheless, most cases are occurred during implantation stage of access device due to blind insertion of catheters into the peritoneal cavity. Of note, bowel erosion during the cycle of intraperitoneal chemotherapy or even after completion of intraperitoneal chemotherapy is very rare. Currently, there exist very few published reports of such cases. Here we report a case diagnosed with stage III epithelial ovarian cancer, who underwent intraperitoneal chemotherapy following primary cytoreductive chemotherapy. This patient had an uneventful life during the first four cycles of intraperitoneal chemotherapy. However, during the fifth cycle of chemotherapy, this patient presented symptoms of watery diarrhea upon infusion of chemotherapy solution. Bowel erosion by catheter was suspected. Infusion of contrast medium into the catheter confirmed that contrast medium flowed into the small bowel. We also conducted a Preferred Reporting Items for Systematic Reviews and Meta-analyses (PRISMA)-driven reviews for published cases.
\end{abstract}

\section{Introduction}

In addition to standard intravenous chemotherapy in the treatment of epithelial ovarian cancer, intraperitoneal (IP) chemotherapy provides a pharmacokinetically advantage of delivering high drug into the peritoneal cavity, a major site for tumor spread. Thus far, three large randomized clinical trials have demonstrated a survival benefit in patients diagnosed with epithelial ovarian cancer who received firstline IP chemotherapy after optimal cytoreductive surgery compared with conventional systemic chemotherapy [1-3].

Furthermore, in long-term follow-up study, IP chemotherapy even shows overall survival benefits that extend beyond 10 years. The longterm survival advantages may encourage more gynecologic oncologist to adopt IP chemotherapy in their communities [4]. Nonetheless, according to a recent report, the adoption of IP chemotherapy is an underused strategy for eligible patients in general, and the integration of IP chemotherapy into clinical practice varies significantly among institutions [5]. Thus, the implementation of IP chemotherapy merits further education and encouragement of gynecologic oncologists.

Although IP chemotherapy confers better survival than conventional systemic chemotherapy. However, toxicities of IP chemotherapy still demand attention. The toxicities of IP therapy are generally related to procedures for administration and/or are drugrelated, as in the case of postoperative therapy [6]. Administration of IP therapeutic agents requires the setup of access devices (e.g., Tenckhoff catheters or Port-A-Cath), the placement, malfunction and adverse effects of which may cause significant suffering to patients, and may introduce delay, prevent the completion of IP chemotherapy [7].

Erosion into adjacent viscus by peritoneal access device during IP chemotherapy is an unusual complication. The presenting symptoms span from minor abdominal pain, watery diarrhea upon IP fluid administration, and even fever and peritonitis. In this article, we report on a case diagnosed with advanced stage ovarian cancer who received IP chemotherapy. During the cycle of IP therapy, patient presented with abdominal pain and watery diarrhea upon IP fluid administration. Treatment and outcome were reported. Also, a systematic review was conducted to identify published relevant reports.

\section{Case report}

Patient KS, is an 86-year-old Asian female with an initial diagnosis

Correspondence to: Chi-Mu Chuang, Institute of Clinical Medicine, School of Medicine, National Yang-Ming University, Taipei, 112 Taiwan (ROC), Tel: +8862-2875-7566, Fax: +886-2-7723-2788, E-mail: cmjuang@gmail.com

Key words: ovarian cancer, bowel erosion, intraperitoneal chemotherapy, tenchkoff catheter

Received: February 29, 2016; Accepted: March 25, 2016; Published: March 28, 2016 
of prominent ascites. First she presented to a gastroenterologist at municipal Zen-I hospital in Taipei for medical assistance in December 2014. Computerized tomography imaging study revealed prominent ascites and multiple nodularity over greater omentum and mesentery, implying carcinomatosis. Upper gastroenteroscopy and colonoscopy revealed non-specific findings. KS was referred to a gynecologic oncologist at Department of Obstetrics and Gynecology in Taipei Veterans General Hospital for further consultation due to high suspicion of pelvic cancer with carcinomatosis.

KS was diagnosed with papillary serous peritoneal carcinoma stage IIIc at primary cytoreductive surgery in January 2015. During cytoreductive surgery, two Tenckhoff catheters were implanted with intent for following intraperitoneal (IP) chemotherapy. Dosing schedules of IP delivery comprised of IP delivery of carboplatin (at AUC=4) with intravenous delivery of Taxol ${ }^{\mathbb{Q}}$ (dosed at $175 \mathrm{mg} / \mathrm{m}^{2}$ ) every three weeks for a total of six cycles. KS lead an uneventful life during each cycle of IP chemotherapy except commonly seen toxicities of therapeutic agents, including muscle pain and numbness. However, ECOG performance score was kept at 0 .

During fifth cycle of IP chemotherapy was delivered in April 2015. KS showed signs of copious watery diarrhea and abdominal cramping pain during IP fluid infusion. Infusion procedure was stopped and pain killer was administered. A fistula between Tenckhoff catheter and bowel was highly suspected. Fluoroscopic image showed contrast medium entering into a loop of small bowel (Figure 1), consistent with erosion of catheter into small bowel. Exploratory laparotomy was performed with consultation of a surgeon. On opening into the abdomen, a Tenckhoff catheter was identified, with tip eroded into a segment of ileum (Figure 2). Catheter was removed with primary closure of the bowel wall defect. A Jackson-Pratt drain tube was placed close to the closure site. KS was recovered completely after operation.

Later on, KS received two cycles of intravenous chemotherapy which was completed in July 2015. In February 2016, all follow-up imaging studies and serum CA125 showed normal readings.

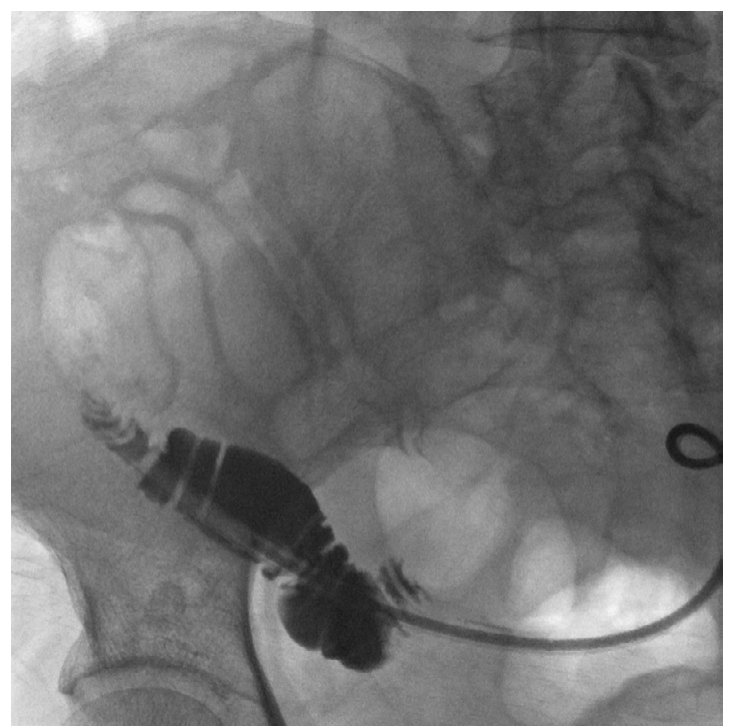

Figure 1. Fluoroscopic image showed contrast medium entering into a loop of small bowel.

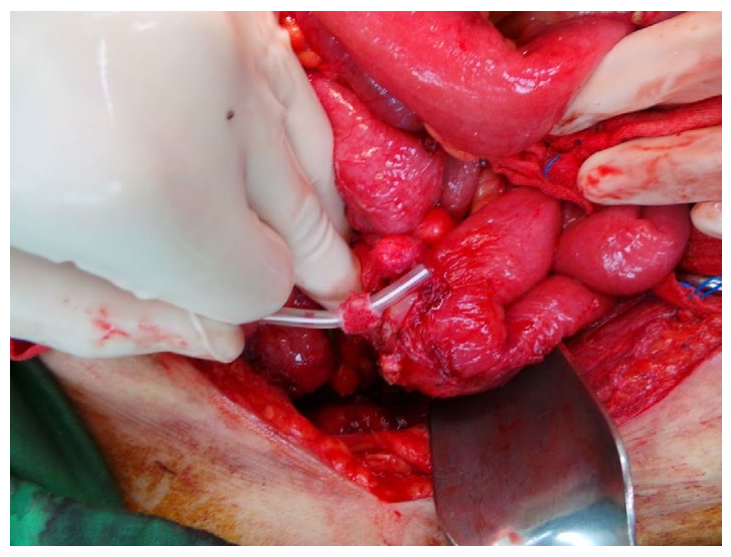

Figure 2. Upon entering the abdomen, erosion into a segment of small bowel by Tenchkoff catheter was shown.

\section{A PRISMA-driven systematic review for bowel erosion during treatment with intraperitoneal chemotherapy}

\section{Methods}

Database retrieval: Electronic database from a total of 1,248 primary epithelial ovarian cancer were retrieved, which is a stably constructed registration system of consecutively treated patients of ovarian cancer, set up in Taipei Veteran General Hospital between January 1985 and December 2015. All information was collected under protocols approved by the hospital Institutional Review Board.

Search strategy of systematic reviews: An extensive literature review was conducted, according to the guidelines proposed at the PRISMA statement [8]. A comprehensive computerized systemic review of published reports, including cancer registry database, randomized controlled trials, cohort studies, and studies of surgical specimens, was performed by searching the following databases: Medline, EMBASE, Cancerlit, Cochrane Database of Systematic Reviews, Cochrane Central Register of Controlled Trials, ISI Web of Science, and Google Scholar. The key search terms included 'ovarian cancer,' 'colon cancer,' 'gastric cancer,' 'Port-A-Cath,' 'Tenckhoff,' 'intraperitoneal chemotherapy,' in combination with the following terms: 'erosion,' 'bowel,' and 'viscus,' The search was limited to human studies published in English from January 1960 to January 2016.

Screening and data extraction: The systematic search described above was completed by January, 2016. Two independent reviewers (H.H.L and C.M.C) assessed the potential relevance of all titles and abstracts identified from the electronic searches. Full articles were retrieved for further assessment when the abstracts indicated that they might meet the inclusion criteria.

The reviewed data were extracted and entered on to an ad hoc standardized data entry form by each reviewer. Data extracted for comparison included study of origin (continent/country), year of publication, research design, number of cases for each subtype, length of recruitment period, source of information.

Assessment of methodological quality: Quality of observational studies (e.g. database analysis, case report, cohort study, and surgical archives) were scored according to the Newcastle-Ottawa Quality Assessment Scale which ranges from 1 (poor) to 9 (excellent) [9]. Because there are no descriptive anchors for this scale except the lowest and highest score, we decided to classify studies with a total score equal to or greater than 7 as high-quality studies. 


\section{Results}

Our search provided a total of 46 citations. After reviewing the title and abstract 35 were discarded for not meeting the criteria proposed. A total of 11 papers were identified for review, as illustrated in PRISMA flow diagram (Figure 3).

Clinical characteristics of 11 included papers [10-20], associated with our case report, were summarized (total case $\mathrm{n}=14$ ) (Table 1).

Among the 14 cases, 11 (78.6\%) were diagnosed with ovarian cancer, while the other 3 cases were diagnosed with colon-rectal or gastric cancer. Most IP infused agent was platinum or platinum combined with other agents (e.g., 5-fluorouracil, mitoxantrone, or taxol). 11 cases (78.6\%) were implanted with Port-A-Cath for peritoneal access, while the other 3 cases were implanted with Tenckhoff catheters. Small bowel is the commonest site of erosion $(n=10)$, followed by colon/rectum $(n=3)$ and ileo-cecal junction $(n=1)$. Treatment for each cases varied, but most cases required removal of catheters with local drainage.

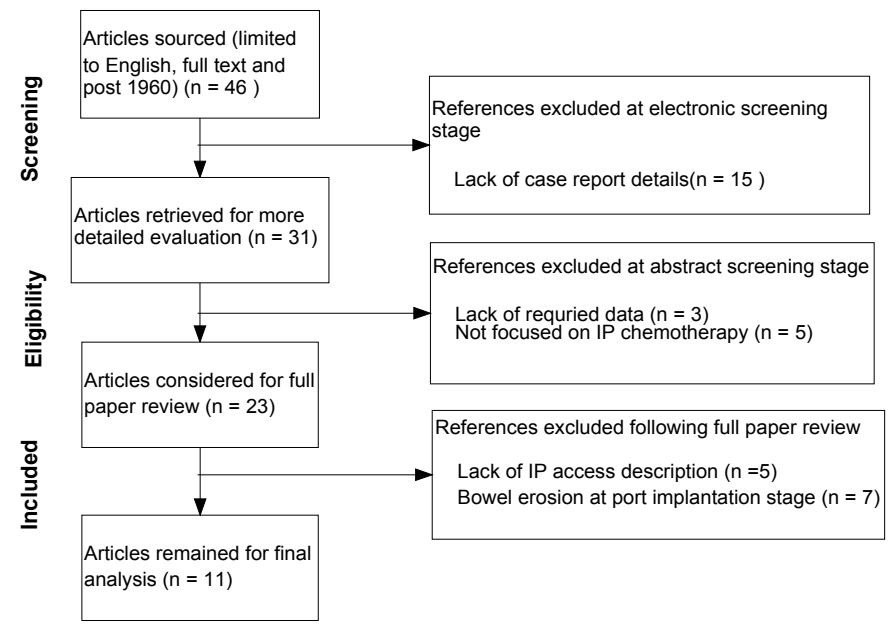

Figure 3. PRISMA flow diagram.

Table 1. Characteristics of included papers $(\mathrm{N}=11)$ and the current study.

\begin{tabular}{|c|c|c|c|c|c|c|c|}
\hline Author & $\begin{array}{c}\text { Year of } \\
\text { publication }\end{array}$ & Disease & IP infused drugs & $\begin{array}{l}\text { Type of peritoneal } \\
\text { access }\end{array}$ & Site of erosion & Treatment & Prognosis \\
\hline Wakfield et al.10 & 1984 & colon cancer & NA & Tenckhoff catheter & colon & Drainage of local abscess & good \\
\hline Holt et al. [11] & 1989 & cecum cancer & 5-fluorouracil & Tenckhoff catheter & sigmoid colon & Removal of Tenckhoff catheter & good \\
\hline Varney et al. [12] & 1989 & & & & & & \\
\hline case 1 & & ovarian cancer & cisplatin & Port-A-Cath & small bowel & $\begin{array}{l}\text { Jackson-Pratt drain with } \\
\text { hyperalimentation }\end{array}$ & good \\
\hline case 2 & & gastric cancer & 6-thioguanine & Port-A-Cath & small bowel & $\begin{array}{l}\text { Removal of Port-A-Cath without } \\
\text { laparotomy }\end{array}$ & good \\
\hline Davidson et al. [13] & 1991 & & & & & & \\
\hline case 1 & & ovarian cancer & cisplatin +5 -fluorouracil & Port-A-Cath & small bowel & $\begin{array}{l}\text { Removal of Port-A-Cath without } \\
\text { laparotomy }\end{array}$ & good \\
\hline case 2 & & ovarian cancer & cisplatin +5 -fluorouracil & Port-A-Cath & small bowel & $\begin{array}{l}\text { Removal of Port-A-Cath without } \\
\text { laparotomy }\end{array}$ & good \\
\hline Topuz et al. [14] & 2000 & ovarian cancer & cisplatin + mitoxantrone & Port-A-Cath & small bowel & NA & NA \\
\hline Sakuragi et al. [15] & 2000 & ovarian cancer & cisplatin & Port-A-Cath & small bowel & Laparotomy with removal of catheter & NA \\
\hline Bilsel et al. [16] & 2005 & ovarian cancer & cisplatin & Port-A-Cath & rectum & Laparotomy with removal of catheter & good \\
\hline Landrum et al. [17] & 2008 & ovarian cancer & cisplatin & Port-A-Cath & $\begin{array}{l}\text { ileo-cecal } \\
\text { junction }\end{array}$ & exploratory laparotomy & NA \\
\hline Berry et al. [18] & 2009 & ovarian cancer & cisplatin + taxol & Port-A-Cath & small bowel & exploratory laparotomy & mortality \\
\hline Lesnock et al. [19] & 2010 & ovarian cancer & cisplatin + taxol & Port-A-Cath & small bowel & NA & NA \\
\hline Kerwin et al. [20] & 2015 & ovarian cancer & cisplatin & Port-A-Cath & small bowel & resection of eroded bowel & good \\
\hline Chuang et al. & current study & ovarian cancer & cisplatin & Tenckhoff catheter & small bowel & primary repair of eroded bowel & good \\
\hline
\end{tabular}

NA: Not Available

Outcome is good for most cases except one mortality case.

\section{Discussion}

During the treatment of IP chemotherapy, erosion into the bowel by catheter of peritoneal access device is relatively uncommon. Most of the reported cases were occurred during the placement or implantation stage of device, and the occurrence rate ranges from $1.2-4.2 \%$, dependent on type of device [21-23]. One study has suggested that placing the catheter while simultaneously undergoing laparotomy and bowel resection predisposes to catheter erosion into the bowel [24].

In contrast, erosion into bowel during the cycle of IP therapy or even after completion of IP therapy is even rarer. In this PRISMAdriven systematic review, we identified 13 published cases in the literature. All these cases presented bowel erosion during the cycle of IP therapy or even occurred after completion of IP therapy. The shortest duration is just three weeks following implantation of access device [10] whereas the longest duration is 36 months following implantation of access device [15]. Our reported case was found to have small bowel erosion at 3 months following implantation of access device.

The exact mechanism of catheter-induced bowel erosion remains unknown due to rarity of reported cases. Potential contributing factors may comprise IP infused agent, solution for infusion, disease status, comorbidities, and structure of bowel. The major characteristic presenting symptom is watery diarrhea upon infusion of IP solution. According the published reports and our case, most cases will have an uneventful outcome without major sequelae. Of note, research has found that the incidence of catheter-related complications appeared to be correlated with the length of time elapsed after initial implantation of access device. As such, investigators have advised to remove catheters within one year after completion of IP therapy [15,17].

In conclusion, although IP chemotherapy has been proved to confer better overall survival in the treatment of ovarian cancer, however, the complications of IP therapy still demand attention. Clinicians have to be alert when a patient shows symptom of watery diarrhea upon infusion of IP solution, the possibility of catheter erosion into bowel need to be suspected. Although the outcome is favorable for most 
treated cases, however, mortality case has been reported.

\section{Funding resource}

This work was supported by the National Science Council, Taiwan (Grants No. MOST 105-2623-E-010-003 -NU).

\section{References}

1. Alberts DS, Liu PY, Hannigan EV, O'Toole R, Williams SD, et al. (1996) Intraperitoneal cisplatin plus intravenous cyclophosphamide versus intravenous cisplatin plus intravenous cyclophosphamide for stage III ovarian cancer. N Engl J Med 335: 19501955. [Crossref]

2. Markman M, Bundy BN, Alberts DS, Fowler JM, Clark-Pearson DL, et al. (2001) Phase III trial of standard-dose intravenous cisplatin plus paclitaxel versus moderately high-dose carboplatin followed by intravenous paclitaxel and intraperitoneal cisplatin in small-volume stage III ovarian carcinoma: an intergroup study of the Gynecologic Oncology Group, Southwestern Oncology Group, and Eastern Cooperative Oncology Group. J Clin Oncol 19: 1001-1007. [Crossref]

3. Armstrong DK, Bundy B, Wenzel L, Huang HQ, Baergen R, et al. (2006) Intraperitoneal cisplatin and paclitaxel in ovarian cancer. $N$ Engl J Med 354: 34-43. [Crossref]

4. Tewari D, Java JJ, Salani R, Armstrong DK, Markman M, et al. (2015) Long-term survival advantage and prognostic factors associated with intraperitoneal chemotherapy treatment in advanced ovarian cancer: a gynecologic oncology group study. $J$ Clin Oncol 33: 1460-1466.

5. Wright AA, Cronin A, Milne DE, Bookman MA, Burger RA, et al. (2015) Use and Effectiveness of Intraperitoneal Chemotherapy for Treatment of Ovarian Cancer. J Clin Oncol 33: 2841-2847. [Crossref]

6. Wenzel LB, Huang HQ, Armstrong DK, Walker JL, Cella D, et al. (2007) Gynecologic Oncology G. Health-related quality of life during and after intraperitoneal versus intravenous chemotherapy for optimally debulked ovarian cancer: a Gynecologic Oncology Group Study. J Clin Oncol 25: 437-443. [Crossref]

7. Helm CW (2012) Ports and complications for intraperitoneal chemotherapy delivery. BJOG 119: 150-159. [Crossref]

8. Moher D, Liberati A, Tetzlaff J, Altman DG; PRISMA Group (2009) Preferred reporting items for systematic reviews and meta-analyses: the PRISMA statement. Ann Intern Med 151: 264-269, W64. [Crossref]

9. Wells G, Shea B, O'connell D, Peterson J, Welch V, et al. (2016) The Newcastle-Ottawa Scale (NOS) for assessing the quality of nonrandomised studies in meta-analyses. [http://www.ohri.ca/programs/clinical_epidemiology/oxford.asp]. Accessed February

10. Wakefield T, Eckhauser F, Strodel W, Knol J (1984) Colocutaneous fistula complicating Tenckhoff catheter placement for intraperitoneal chemotherapy. J Surg Oncol 27: 205207. [Crossref]
11. Holt RW, Petrilli ES, Evans SR, Ahlgren JD (1989) Intraperitoneal chemotherapy complicated by erosion of a Tenckhoff catheter into the colon. South Med J 82: 914 916. [Crossref]

12. Varney RR, Goel R, vanSonnenberg E, Lucas WE, Casola G (1989) Delayed erosion of intraperitoneal chemotherapy catheters into the bowel. Report of two cases. Cancer 64: 762-764. [Crossref]

13. Davidson SA, Rubin SC, Markman M, Jones WB, Hakes TB, et al. (1991) Intraperitoneal chemotherapy: analysis of complications with an implanted subcutaneous port and catheter system. Gynecol Oncol 41: 101-106. [Crossref]

14. Topuz E, Salihoglu Y, Aydiner A, Saip P, Tas F, et al. (2000) Celsite port and catheter as an intraperitoneal access device in the treatment of ovarian cancer. J Surg Oncol 74 223-226. [Crossref]

15. Sakuragi N, Nakajima A, Nomura E, Noro N, Yamada H, et al. (2000) Complication relating to intraperitoneal administration of cisplatin or carboplatin for ovarian carcinoma. Gynecol Oncol 79: 420-423. [Crossref]

16. Bilsel Y, Balik E, Bugra D, Yamaner S, Akyuz A (2005) A case of protrusion of an intraperitoneal chemotherapy catheter through rectum. Int J Gynecol Cancer 15: 171174. [Crossref]

17. Landrum LM, Gold MA, Moore KN, Myers TK, McMeekin DS, et al. (2008) Intraperitoneal chemotherapy for patients with advanced epithelial ovarian cancer: review of complications and completion rates. Gynecol Oncol 108: 342-347. [Crossref]

18. Berry E, Matthews KS, Singh DK, Buttin BM, Lurain JR, et al. (2009) An outpatien intraperitoneal chemotherapy regimen for advanced ovarian cancer. Gynecol Oncol 113: 63-67. [Crossref]

19. Lesnock JL, Richard SD, Zorn KK, Krivak TC, Beriwal S, et al. (2010) Completion of intraperitoneal chemotherapy in advanced ovarian cancer and catheter-related complications. Gynecol Oncol 116: 345-350. [Crossref]

20. Kerwin L, Calhoun S (2015) Delayed Intraperitoneal Catheter Erosion into the Small Bowel. Case Rep Radiol 2015: 697608. [Crossref]

21. Jenkins J, Sugarbaker PH, Gianola FJ, Myers CE (1982) Technical considerations in the use of intraperitoneal chemotherapy administered by Tenckhoff catheter. Surg Gynecol Obstet 154: 858-862. [Crossref]

22. Myers CE, Collins JM (1983) Pharmacology of intraperitoneal chemotherapy. Cancer Invest 1: 395-407. [Crossref]

23. Piccart MJ, Speyer JL, Markman M, ten Bokkel Huinink WW, Alberts D, et al. (1985) Intraperitoneal chemotherapy: technical experience at five institutions. Semin Oncol 12: 90-96. [Crossref]

24. Walker JL, Armstrong DK, Huang HQ, Fowler J, Webster K, et al. (2006) Intraperitonea catheter outcomes in a phase III trial of intravenous versus intraperitoneal chemotherapy in optimal stage III ovarian and primary peritoneal cancer: a Gynecologic Oncology Group Study. Gynecol Oncol 100: 27-32. [Crossref]

Copyright: (C) 2016 Lin HH. This is an open-access article distributed under the terms of the Creative Commons Attribution License, which permits unrestricted use, distribution, and reproduction in any medium, provided the original author and source are credited. 